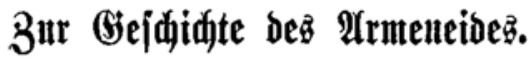

$\mathfrak{B o n}_{\text {on }}$

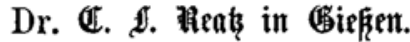

(Fine gründidje Darftellung ber (Seffujidte bes Armeneides im

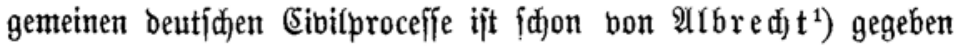

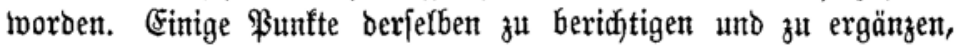

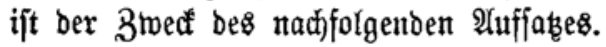

I. Der Armeneid in der italienifhen praxis.

§. 1.

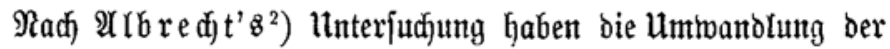

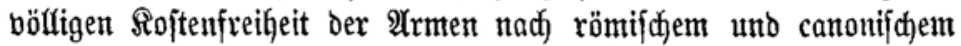
Rechte in eine blop̧e Stundung ber ßroceß̧foften bis zu fünftiger

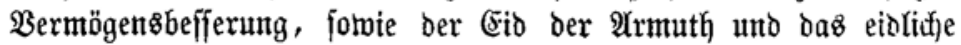
Berfpreçen der Bebührenrefittution bei befferen Bermögensoerhält=

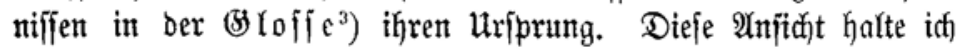
nicht für ridftig. Das Stmoungsred,t und ber $\mathfrak{A}$ rmeneio finten fid fdon früher und in ber italienifden $\mathfrak{B r a r i s . ~}$

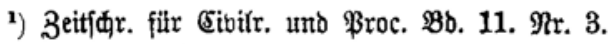

2) a. a. D. S. $90-93$.

3) (II. notabiliter za c. 11. §.4. de rescript. in VIto. (Bonifacius VIII. a. 1302.) „Notabilis igitur debet esse paupertas. ad i dem supra de usur. cum tu." 


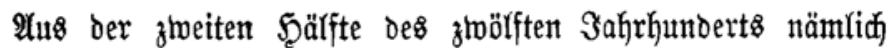

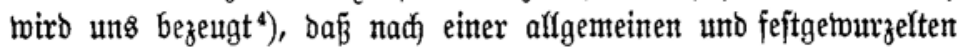
Braxis ${ }^{5}$ ) ber italienifdjen Seridfte bie ftreitenden Theile beim $\mathfrak{B e}=$

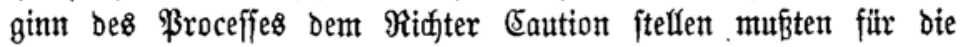
Zahlumg ber Sporteln uno Expenfen, zu weldjer ber unterliegende

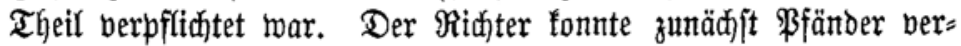
Iangen. War eine Barthie nidjt im Stande, Bfänber zut geben, fo muß̧te fie $\mathfrak{B u ̈ r g e n ~ f t e l f e n , ~ u n o ~ t w a r ~ i h r ~ a u c h ~ b i e s ~ u m m o ̈ g l i c h , ~ f o ~}$ muste fie juratorifde (Saution leiften. Die Behauptung biejes $\mathfrak{U n}_{\mathfrak{n}}=$ bermögens twurbe befojworen. Diejer lestere Eid ijt feinem $\mathfrak{B}_{\text {ejen }}$ nach ber (Fio ber $\mathfrak{A}$ rmuth. Das eidlidje QBeriprecten ber Sporteln= uno Expenfenzahlung war jicherlich nidjt nur bon ben $\mathfrak{B}$ erlujt bes

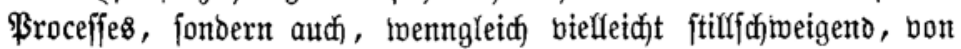
ber Bahlungsfähigfeit, o. h. vom Eintritt befferer als ber gegen= twärtigen Bermögenşberhältniffe bebingt. ${ }^{6}$ ) Şieraus ijt benn auch

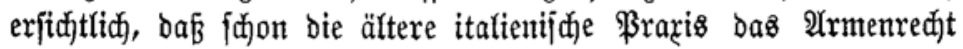
als Stundungorecht fannte.

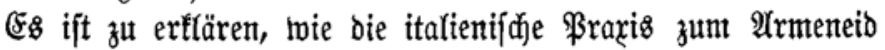
gelangte.

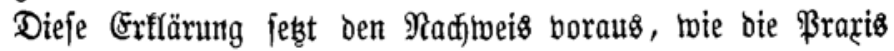

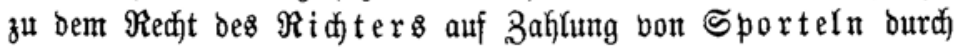
ben Unterliegenden, und auf Stellung einer $\mathcal{S}_{\text {aution }}$ burch beibe Barthieen beim Beginn des Procefies ges fommen ijt.

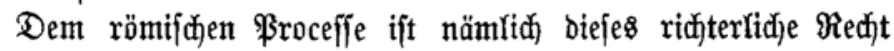
böltig fremb. Der römifje Magiftrat fann feine Sporteln for = bern. Nur jeine Dfficialen, Sanzlei= uno Exrecutibbeamten erhalten

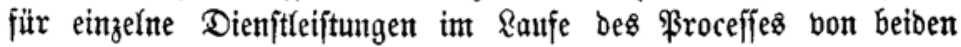
ßartfieen Sporteln. ${ }^{7}$ ) Der judex pedaneus empfängt, wenn bie

4) Johannes Bassianus, Summa "Quicunque vult". 2 bgebruđt

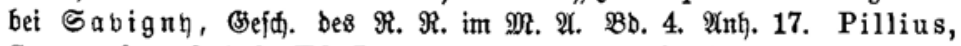
Summa de ord. jud. (Ed. Bergmann) p. 1. §. 1. ङ. 5.

5) $\mathfrak{B a n n}$ fie entiftant, Iäßjt fidh niḑt beftimmen. Sie exhielt fith aud mährend des breizehnten Safrhunbert8. Tancredus, ord. jud. (Ed. Bergmann) p. 2. t. 15. §. 1. S. 176. Durantis, spec. jur. 1. 2. p. 1. de satisdationibus. §. 2. n. 2. Beibe jebod mit Berufung auf Pillius.

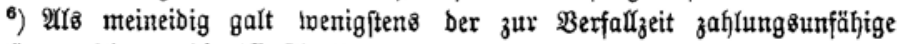
Sđaulbner nidt. c. 10. 17. X. de jurejur. (2. 24).

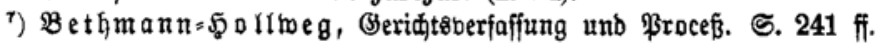


Sacje ben $\mathfrak{B e r t h}$ bon hunbert 2 lurei überjteigt, bei ber Ritis: conteftation uno beim (Enourtheil ie zwei Arurei. ${ }^{8}$ ) Dieje Sportern haben bie Natur einer $\mathfrak{B}$ ergütung für bie bon ben genannten Per= foren ben Parthieen geYeijteten Dienjte.

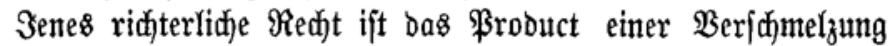

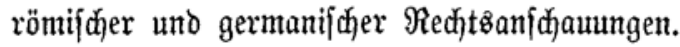

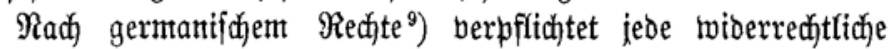
Şandlung zun Exfags bes aus ihr entítandenen Sdjabens; biele

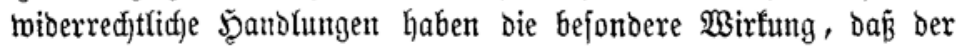

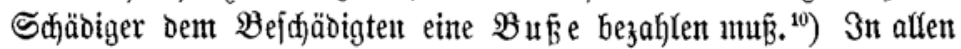
Fäflen, too eine $\mathfrak{B a r t h i e ~ i h r e m ~ S t e g n e r ~ e i n e ~} \mathfrak{B} u \tilde{B} e$ zu zahlen hat,

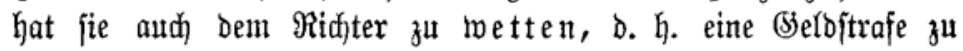

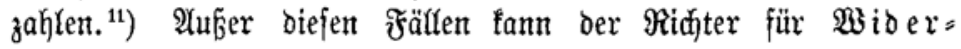

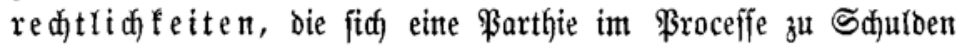
fommelt läßjt, bie $\mathfrak{B e t t e}$ fordern. ${ }^{12}$ ) Der gemeinfame (5yaracter

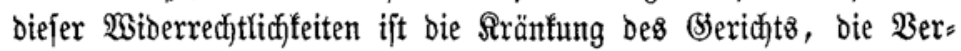
lekzung ber richterlichen $\mathfrak{B u ̈ r b e}$. Daher trirb ein Strafgeld be= zahlt, wenn eine Barthie zum angejebsten Ternin gar nidjt ober 子u (pät fommt ${ }^{13}$ ), lvenn (Einer ein Urtheil fajilt uno unterliegt ${ }^{14}$ )

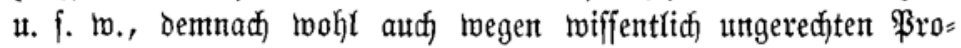
ceffitrent ${ }^{15}$ ), twährend twegen des $\mathfrak{B e r l u j t s ~ d e z ~ \$ r o c e f f e s ~ a l l e i n ~ b e m ~}$ Ridjter feine Wette gebüfrt. ${ }^{16}$ )

8) Bethmann= Forrmeg, a. a. D. S. 147. 243.

9) Reiber find nid,t alle in ber folgenden Darftellung enthaltenen Bunfte

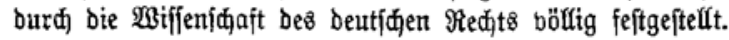

10) Siegel, Befdidite des beutijen Beridtsverfahrens. Bb. 1. §. 23,

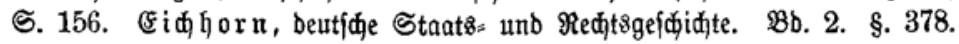

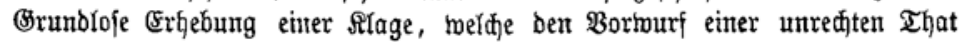
einffifief̧t. 1. Sal. tit. 21. l. Rib. tit. 38. tit. 41. 1. l. Baiuv. c. 8 .

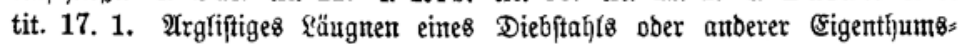
bejđübigungen. l. Sal. tit. 10. 4.

11) Siegel, a. a. D. S. 157. (idhorm, a. a. D. §. 385. Dot erbliatt Sieger in ienem Brelde tein Straf= fonbern ein Friebenzgelb. S. baj. und §. 3. S. 26.27.

18) Eidyorn, a. a. D.

13) Säфf. Lanbr. B. 1. Irt. 53. 28. 2. भrt. 6.

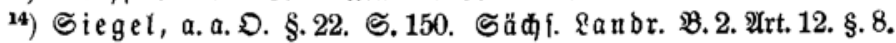

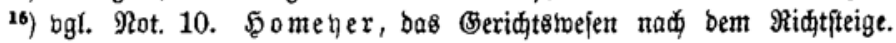

§. 10. ङ. 437. Beile 12. v. o.

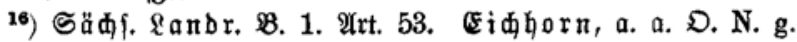


Beim Beginn Des \$roceffes fann Der Richter bon beiden Theilen ${ }^{5}$ aution berfangen, $b a \tilde{B}$ fie in ben angefesten Terminen

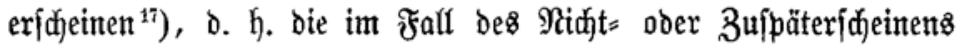
berwirfte Bette bezahlen würden. Ferner muâte jebesmal nad bem Berfalf ber Wette Caution für beren rechtzeitige 3 ahlung geleiftet werdent. ${ }^{18}$ )

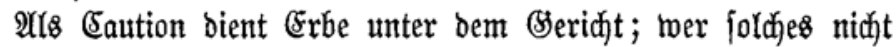
hat, fteflt $\mathscr{B a ̈ r g e n .}{ }^{19}$ )

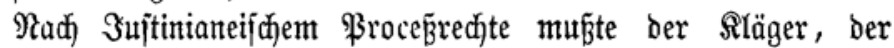

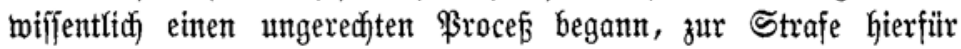
Dem Beflagten ben zel)nten Theil des cingeflagten $\mathfrak{B}$ erthes (decima parș) zahlen, unt für biefen Fall beim Beginn bez ßroceffes (Eaution mittelịt Bürgen (telfen. ${ }^{21}$ )

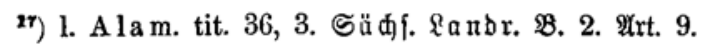

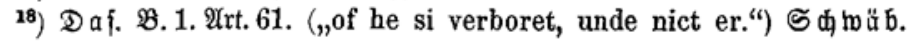
\&anbr. (\&aß̧b.) c. $117 \mathrm{~b}$.

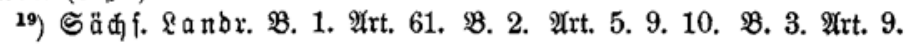
10. 11.

so) Nov. 112. c. 2. pr. Ad excludendas autem calumniose moventium intentiones et executorum fraudes, aliud invenit providentia nostra remedium. Sancimus enim, omnes judices - hanc conditionem in suis interlocutionibus adjicere, ut non aliter conventionales libelli pulsatis porrigantur, aut sportulae executoribus dentur, nisi prius et in libello actor per se aut per tabularios subscripserit: et actis intervenientibus fidejussorem idoneum - praestiterit - : et si postea fuerit approbatus injuste litem movisse, sumptum et ex pensarum nomine decimam partem ejus quantitatis, quae in libello continetur, pulsato restituat. Der decima pars legte id obent Den (styarafter eines Strafgelbez bei. Diefe Befjauptung bedarf jebod)

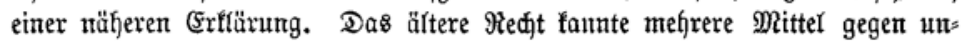
gereçtes \$roceffiren, u. a. bas Calumniae judicium, auf toeldę ber Beflagte einen $\mathfrak{2}$ (p)

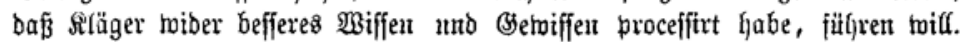

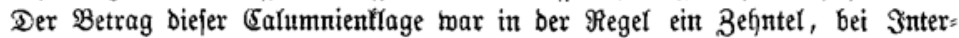
Dicten ein Siertel Des Štreitgegenftandeళs. Gai us l. 4. §. 175. 178. 163. Cod. Hermog. V, 3. Der Eharacter biefer Summen mar ber eines reinen Strafgeldes, fo Daß̧ bie bom Beflagten aufgemendeten ßroceß̧foften nebenbei bezahłt merben mußjten. Affein biefes Calumniae judicium fdeint in ber

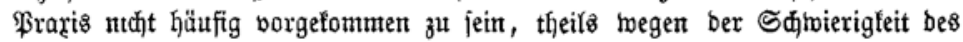

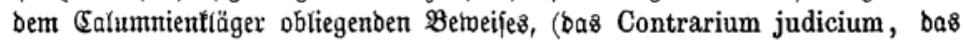
jebn(t) nur ex certis causis gegebent twirb, mar bortheilf(fafter, meil mit ber 


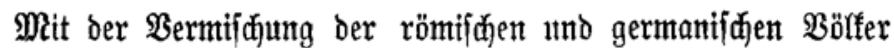

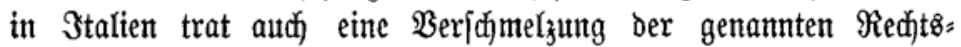
anjdjaunngen beider $\mathfrak{B o ̈ l f f e r ~ z u ~ e i n e r ~ n e u e n ~ e i n . ~}$

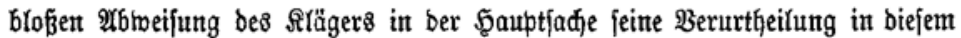
judicium eintrat,) theils meil nod anbre, bielleidt twirffamere Sđjutzmittel

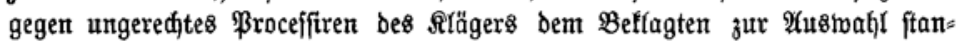
ben, 3. B. bas Jusjurandum calumniae. Gaius, 1. 4. §. 172. inßbef. §. 178 ff. 3u $3 u f t i n i a n ' \&$ Beit fam bas Calumniae judicium gar nidjt

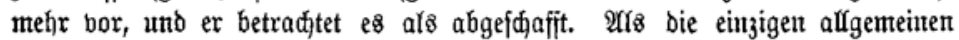
Sđjutzmittel gegen ungeređtes Broceffiten fitfyt er ben Salumnieneid und bie

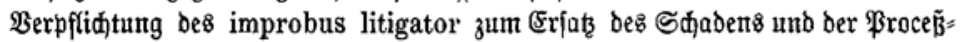
foften Des (3egners auf. §. 1. J. de poen. tem. litig. (4. 16). Später jeboch fah er bie Roth́twendigfeit einer bejonderen Beftrafung der Calumnia

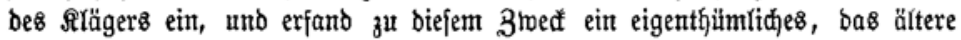
und nentere Red,t berfamelzendes Mittel. Der calumniofe Rläger foll ben zelpnten Tfyeil bes Streitgegenftandes sumptuum et expensarum no-

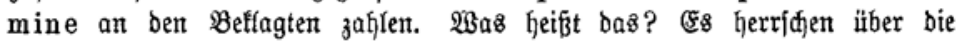
Bebeutung biefer decima pars beriđjiedene $\mathfrak{A} n$ fidjten. Unbeftimmt brifft fid

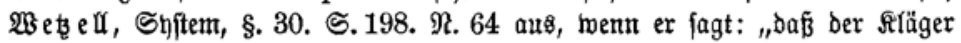
- wemn fid ber Ungrund feiner Rlage herausftelle, Den 10. Theil Des Streit= objects in Betreff ber $\mathfrak{R}$ often un $\mathfrak{A} u$ \&lagen Dem Berflagten zahlen

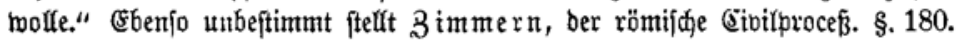
$\mathfrak{N}$. 21 bie Sache bar: „In Nov. 112. c. 2 ift aud mieber bon einer bem (5)gner zur (Entfđäbigung zuzufiđjernden decima pars beళ Streitobject\& die Rebe. f. §. 181." Diefe Unbeftimmtheit mirb jebod in bem cit. §. niđt ges hoben. Bethmann= Follweg, Beridtsberfaffing unt ßroceß̧, §. 22. S. 255 und $\mathfrak{B a h e r , ~ B o r t r a ̈ g e , ~ § . ~ 3 3 . ~ S . ~} 99$ findent in ber Nob. ausges fprođjen, baß̧ der siräger eine den zehnten Theil bes Streitobjects betragende Eaution für bie 3ahlung ber \$roceßftoften ftellen müfife. Daraus toïrbe folgen,

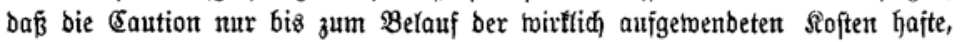

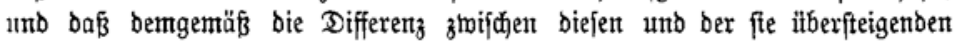

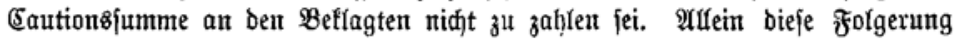

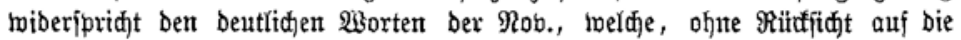
Bröfe ber in einem Froceffe aufgemendeten אoften, bie Zahlung ber decima boridreibt. Şeffter, Suftem, §. 275 und Sclaner, bie Rehre bon ben

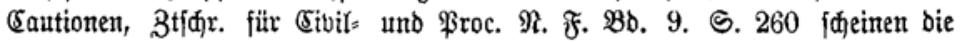
decimae litis als 2 (berfionalfumme für alle $\mathfrak{R}$ often, fie mögen jente itberfteigen

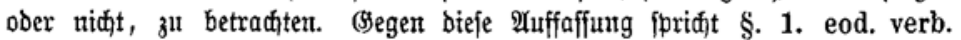
Omne autem dispendium, und Nov. 53. c. 1. 2., tro troţ beftinumter Eau=

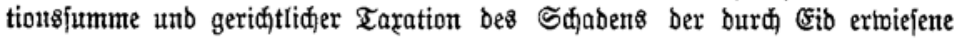
Mehrbetrag bes Sdjabens nadgeforbert werben fann. M. $\mathfrak{A}$. nach hat bie decima litis ben Eharacter bes Roftenerjatzes unb Des Strafgeldes zugleid. Sint im einzelnen falf bie Soften höher als bie decima, ober ihr gleiđf, bann 
Bon germanifden Rechtsgedanfen erhielt jid. :

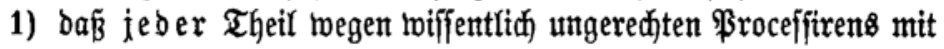

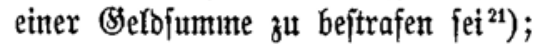

2) Daß̧ bas Strafgelo bem $\Re$ idter gebühre;

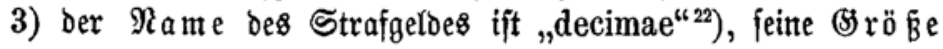
meijtens ber zehnte $\mathfrak{T h e i l}^{23}$ ) Des eingeflagten $\mathfrak{W e r t h e s , ~ m i e ~ i m ~}$ römifden Redite;

4) Den (Stedanfen beider Redte, baß̧, mem bas Strafgeld zu= fomme, Saution für beffen Zahlung fordern fönne, behielt man bei, unb ba es in ber neuen $\mathfrak{B r a r i s}$ an ben $\Re$ idjter zu bezahlen war, fo fonnte folglid er beim $\mathfrak{B e g i n n}$ bes Broceffez (5 a uti on bon beiben Theilen berlangen.

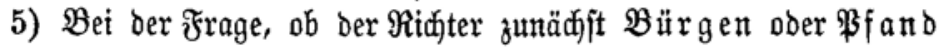
verlangen folle, gab man bem germanifacen Red te ben

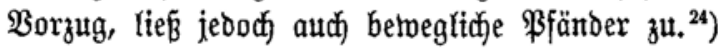

bleibt ber caluntutiofe SYäger ohne Strafe; find fie geringer als jente, bam tritt bie decima als Strafgeld herbor. Trotz ihres (Sharacters als Sqabens: erfał aber twirb fie bon ber Befetzgebung in Banzen als Strafgeld be= zeiđnet, c. 2. cit. c. 5. Edict. Justin. 7. „decimae partis poenam",

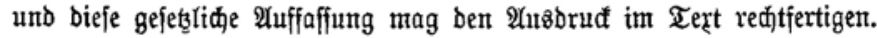

21) Den geftändigen Sdultbner traf fonad) bie Strafe nidt. Dies erfent an\&brïđflid an bas \$ribileg Şeinriđ III. für Ferrara (1055): Preterea si quis ad placitum vocatur, et debitum suum sponte confitetur, a pena Tertiarum solutus sit. cf. Mu rat o ri, Antiquit. med. aev. V, 5. 753.

22) ßirliu \&, a. a. D. p. 1. §. 1. S. 6.

23) Form. ad Leg. Longob. Guid. c. 3. bei Walter, Corp jur. germ. tom. III. (Poena est appellatoris quidem decima pars litis aliquando, et appellati victi.) So ferner in Bologna. Azo, summ. in Cod. lib. 3. de sport. et sumpt. n. 7. Durantis, a. a. D. 1. 1. p. 4. de salariis. §. 1. n. 9. - Joh. Andreae bemerf́t hierzu, bafi zu feiner 3eit bie decima gar nidjt, und frïher nur bon ben Forenfen geforbert morben twäre.) $\mathcal{U}$ andern Drten zafite man bie tertia (cf. Pote 21. Azo, a. a. D.), an andern bie vigesima (Azo, Durantis, a. b. a. D.), bie quadragesima (Azo, a. a. S.), in terris Comitissae Mathildae bie medietas (Durantis, a. a. D.), im patrimonium beati Petri und in ber provincia Romaniola turben 3 tölf denarii de libra gegeben. (Durantis, a.

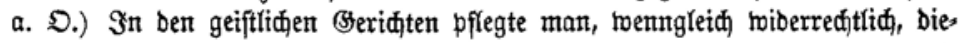

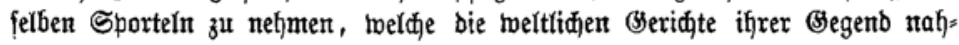
men. c. 10. X. de vit. et hon. cler. (3. 1).

24) Joh. Bassianus, a. a. D. verb.: Sed quia irrepsit utilitas pignorum. Pillius, a. a. D. p. 1. §. 1. Tancredus a. a. D. p. 1. tit. 15. §. 1 . 
Die $\mathfrak{B}$ edeutung ber decima ałs eines Strafgelbes für wif =

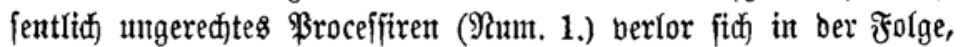
und man begann, fie als eine Bergütung für bie vom Ricter ben Barthieen geleifteten Dienjte, als Sporteln anzulehen, ohne jeboch bie neute $\mathfrak{A n}$ fdauung confequent burcfzuführen. So berlangte man nidłt ettwa bon jebem Theil die ફ̧älfte, fondern ber Sieger war fportelfrei, und ber Unterliegende mup̧te bem Riduter bie decima bezahlen.

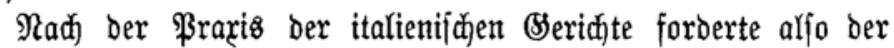
Ridhter beim $\mathfrak{B}$ eginn bes Broceffę bon beiben Theilen $\mathfrak{B}$ fänber für bie Zahlung ber Sporteln. Was joflte geffehen, ment eine Barthie behauptete, leine Pfänder gebent zu fönnen? Für biefen

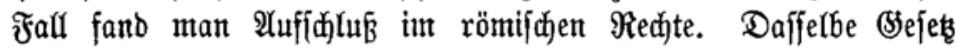

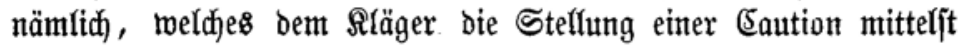
Bürgen wegen ber an ben Beflagten zu zahlenden decima befieflt,

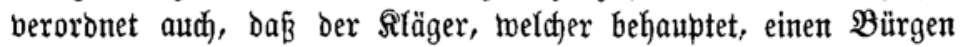
nidjt fteflen zu fönnen, diefes unvermägen eiolid erhärten uno eine juratorifde (5aution leijten folle. Dieje Borfarift

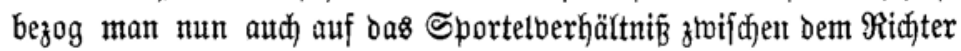
and ben Bartfieen uno gelangte fo zut unferm (E i be ber $\mathfrak{A} r=$ muth und dem eibliden Berfpredfen oer Sporteln= und Expenfenzahlung im Fall befferer Bermögenser= hältniffe. ${ }^{25}$ )

II. Der Xrmencid in Der praţis Der geiflidfen Beridjte.

§. 2 .

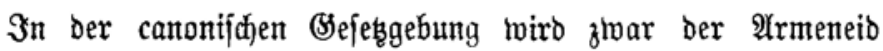

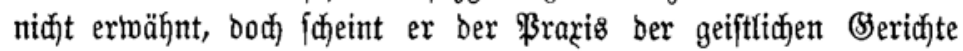

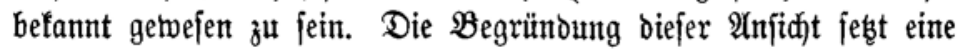
Dariftellung ber Rehre bon ben Bseridgtofojten im canonifden Bros ceffe boraus.

2s) Nov. 112. c. 2. pr. - Si autem dixerit, non valere fidejuss o rem dare: tunc apud judicem, a quo causa examinanda est, sanctis propositis evangeliis, per sacramentum hoc ipsum affirmet, et sic juratoriam cautionem exponat, et per eum haec, quae superius dicta sunt, profiteatur. 
Die Rojten, b. h. bie Belojummen, weldje bie \$arthieen an bie Serichtsperfonen zut zahlen haben, fint theils $\mathfrak{B}$ ergut tungen für bie von biefen geteifteten Dienjte, sportulae, salariae, theil\&

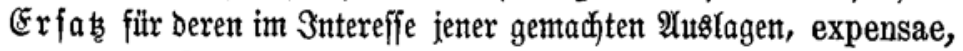
sumptus. Die 和lidft zur Zahlung biefer Roften wiro nidyt nadh berfelben $\Re$ egel beftimmt, es find biełnehr einige Unterfojeibungen zit madjen.

I. Die an ben Ridter za zahlenden Roften.

1) Der geiftlidfe Ridyter, Der orbentlidye wie ber belegirte, foll fitc) mit feinen firchliden Einfümften begnügen, und bon ben Parthicen feine Sporteln nefmen. ${ }^{26}$ ) $\mathfrak{D b}$ bamit aud ber geift=

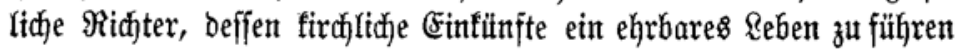
nidjt geítatteten, gemeint fei, toar bejtritten. Aegidius de Fuscararii ${ }^{27}$ ), Tancredus ${ }^{28}$ ) und Gratia ${ }^{29}$ ) (prachen ifm die Sporteln zat, Durantis ${ }^{30}$ ) ab.

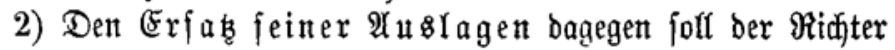

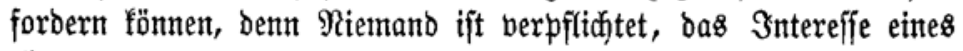

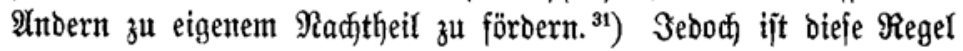

$\left.{ }^{26}\right)$ c. $10 . \mathrm{X}$. de vit. et hon. cler. (3. 1.) c. 71. C. 11. qu. 3. c. 15. C. 14. qu. 5. c. 11. §. 4. de rescr. in VIto. (1. 3). ફ̧iernađ und nad 2.

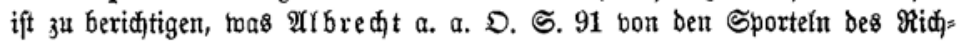

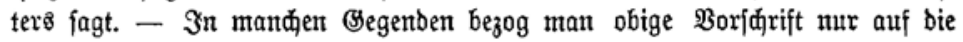
Brälatent. cf. Gratia, de jud. ord. p. 2. t. 9. (Ed. Bergmann. S. 378).

${ }^{27}$ ) Ord. jud. tit. de salario judicum. (Nad) einer $\mathfrak{B}$ a mberger Şand ¡đrift P. II. 2. fol. 17. cap. LVI). Si autem aliquis est pauper clericus, qui non habet de bonis ecclesiae, unde vivat et cognoscat de aliqua causa, si exinde salarium percipiat, non est reprehendendum, nemo enim tenetur propriis"stipendiis militare.

${ }^{28}$ ) Ord. jud. p. 2. t. 15. §. 2. S. 176.

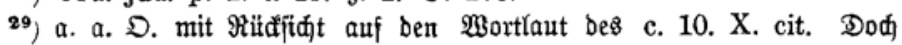

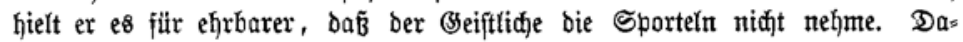
gegen finbet er in ben Bejęzen niđjt verboten, unb baḩer exlaubt, daß̧ ber in nieberen $\mathfrak{B e i f h e n ~ f i t h e n t b e ~ B e i f t l i d j e ~ S p o r t e l n ~ n e h m e . ~ ( c . ~ 7 1 . ~ C . ~ 1 1 . ~ q u . ~}$ 3. c. 15 . C. 14 . qu. 5.)

so) Specul. jur. 1. 1. p. 4. de salariis §. 1. n. 7 in $\mathrm{f}$.

s1) c. 10 . X. cit. (c. 3 . §. 40. C. 4. qu. 3. c. 4. §. 1. C. 10. qu. 2. c. 8 in f. C. 28 . qu. 1. c. 16. X. de praescript. (2. 26.) c. 10. X. de simonia (5. 3.)). Hostiensis erblifte in ber Annahme bes Erfatzes ber $\mathfrak{B} e r=$

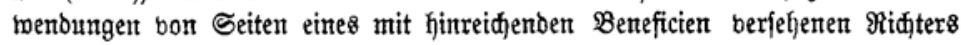
eite Sinbe. cf. Durantis, a. a. D. pr. gl. moderatas zu c.11. §. 4 cit. 
beföräntt auf ben bom apojtolijijen Stuhle belegirten Ridater. ${ }^{32}$ ) Der ordentlidje Ridjter fann ben Erjaks der innerhalb feines $\mathfrak{B e}_{e}$

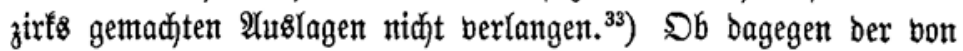
biejem belegirte Richter, war unter ben (Sloffatoren bejtritten. ${ }^{34}$ )

Die $\mathfrak{A}$ ut\&lagen betreffen Rebensunterhalt und Transport. $\mathfrak{A n}_{\mathfrak{n}}$

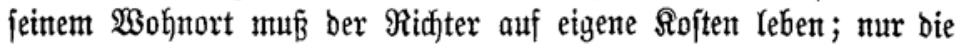
auf ber Reife gemadjten 2 (uslagen werben ihm erfest ${ }^{35}$ ), und fie nur bann, wenn bie Reije burdy bie Sadje ber ßarthieen herbor= gerufen ${ }^{36}$ ), und für biefelben nöthig oder nübslidy ift. ${ }^{37}$ ) Şter aber

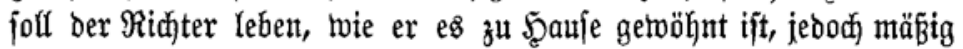

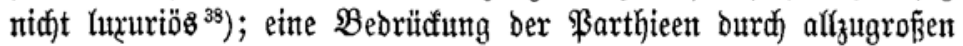
Uuftwand ijt berboten. ${ }^{39}$ ) Man ftritt barïber, ob bie ßarthieen

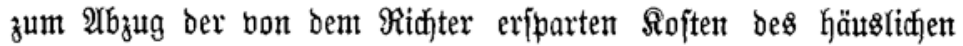
Rebensiunterfalts beredjtigt feien. ${ }^{40}$ )

32) c. 10. X. cit. gl. not. gl. praeter expensas fierzu.

33) Man fürojtete Erpreffungen. c. 5 pr. C. 23 . qu. 1. Gl. not. gl. praeter expensas $z^{\mathfrak{u}}$ c. 10. X. cit. Dem Ordinarius toerben bie $\mathfrak{A}$ (ulagen erferzt, went er eine fitirde feitur ßrobint confecrirt, c. 10. X. de simonia (5. 3) ober leţtere bifitirt. c. 6. 14. 25. X. de censibus (3. 39).

$\left.{ }^{34}\right)$ Berneint bon gl. praeter expensas zu c. 10. X. de vit. et hon. cler. Eine Unterfijeibutg mađen gl. moderatas zu c. 11. §. 4 cit. Du-

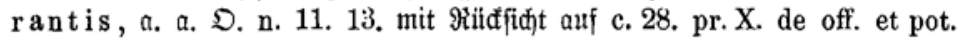

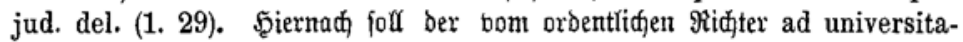
tem causarum, ober ad specialem causam, aber malitiose belegirte Ridter ben Erjat3 ber Bermenbungen niđjt berlangen tömen, weil in beiben Fäflen ber Ordinarius fïr feinen Rebensunterhalt zu forgen habe. Intbers wenn ber

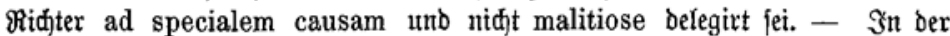
Braris pflegtent bie Delegirten ohne Unterfáieb bie Erpenjen zu forbern. cf. gl. praeter expensas ju c. 10.X. cit. Joh. Andreae ad v. faciant biefer Gl. Durantis, a. a. D. n. 11.

35) c. 11. §. 4. cit.

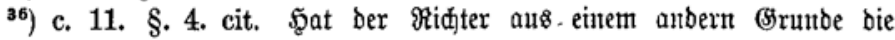

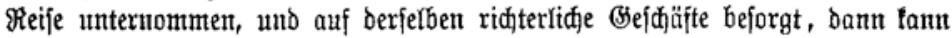

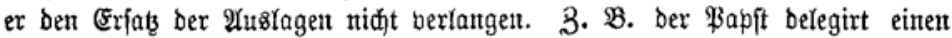
in Paris ober Bologna fids aufhaltenden Sdjolarent. Durantis, a. a. $\mathfrak{D}$. n. 6. gl. propter causam 孔̆ c. 11. §. 4. cit.

${ }^{37}$ ) c. 11. §. 4. cit.

$\left.{ }^{38}\right)$ c. $11 . \$$. 4. cit. gl. moderatas hierzu. Durantis a. a. D. pr.

$\left.{ }^{39}\right)$ c. 11 . §. 4. cit. c. 7. C. 10. qu. 3. Gratia, a. a. D. p. 2 . t. 9. ธ. 379 .

$\left.{ }^{40}\right)$ gl. not. gl. moderatas 3u c. 11 . \$. 4 cit. Durantis, a. a. $\mathfrak{D}$. n. 3. Joh. Andreae, not. g. zu biejer Stelle. 
Die Arslagen werben von ben \$arthieen zu gleidhen Theilen getragen ${ }^{41}$ ), benn ber $\Re$ idjter hantolt jtets im 3 nttereffe beiber ${ }^{2}$ ), mag auth nur eine bie Sornahute bes ausioürtigen (befduäts beranlaß̧t haben. ${ }^{43}$ ) Sift jedoch ber Drt biejes (Sejdäfts bom $\mathfrak{B}_{0}$ hnort bes Riduters toeiter at\& ein $e^{44}$ ) Tagereife $e^{45}$ ) entfernt, Daun hat ber Antragiteller bie Erpenjen allein zut tragen. ${ }^{46}$ ) ऊjt eine Parthie arm, fo hat bie vermögende bie Expenjen alfein zu tragen $\left.{ }^{47}\right)$, und jind fie beibe arm, fo foll fid ber Ridjter auf eigene Sioften unterbalten. ${ }^{49}$ )

II. Die $\mathfrak{A}\left\{f_{\text {efforen }}{ }^{49}\right)$ und $\mathfrak{R o t a r e}^{50}$ ) erfalten Sporteln,

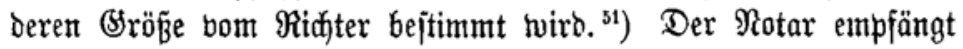

41) c. 11. \$. 4. cit.

$42)$ c. 11 . §. 4. cit. c. 15 . §. 1. C. 14 . qu. 5 .

$\left.{ }^{43}\right)$ Frither beftrittelt. cf. Durantis, a. a. D. n. 2.

44) c. 11. §. 1. de rescr. in VIto. (1. 3.) Früher ztoei Tagereifen. c. 28 . pr. X. de rescr. (1. 3.) c. 7. X. de except. (2. 25). Durantis, a. a. D. n. 2. X. cit.

45) Ueber bie Bedeutung berfelben gl. ultra duas diaetas zu c. 28. pr.

$\left.{ }^{46}\right)$ c. 28 . pr. X. cit. unt gl. ultra duas diaetas hierzu. c. 7. X. cit. unb gl, in tali loco statuerit bierzu. Durantis, a. a. D. n. 2. c. 11. §. 1. cit.

${ }^{47}$ ) Gl. quam ab alia zu c. $11 . \S .4$. cit.

$\left.{ }^{48}\right)$ c. 11. §. 4. cit. gl. casus. gl. not. hierłh. - gl. praeter expensas

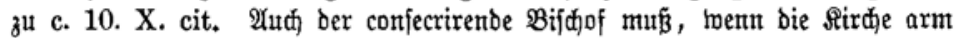
ift, auf eigne Roften leben. c. 1. C. 1. qu. 2, gl. procurationem zu c. 10. X. de simonia (5. 3).

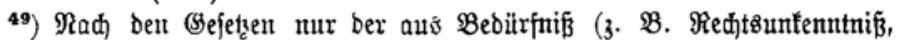

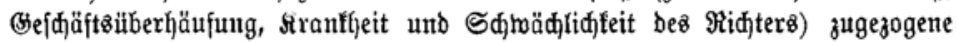

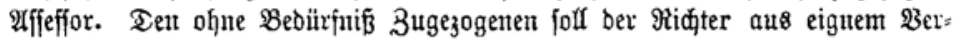
mögen honoriren. Dođ) entidjieb über bie Bebürfnif̧frage Yebiglid bas Betwiffen

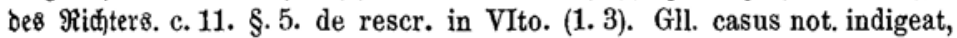
relinquatur. Bor biejer Decretale mar bie Frage, ob ber $\mathfrak{A} f f e f f j o r$ Sporteln neḩmen bürfe, beftritten. cf. Tancredus, a. a. D. p.1. t.4. §.5. S.110. p. 2. t. 15. §. 2. S. 176. Durantis, a. a. D. 1. 1. p. 1. de assessore. §. 5. pr. Gratia, a. a. \. p. 2. t. 9 in f. $\$ .379$.

so) c. 11. §. 6. de rescr. in VIto. Durantis, a. a. D. l. 1. p. 4. de salariis. §. 2. n. 4.

b) c. 11. §. 5. cit. Gratia, a. a. D. p.2. t.9. 5. 379. Durantis, a. a. D. de assessore. §. 5. n. 2. 4. Befđaffentheit ber Sađe, இermögenz:

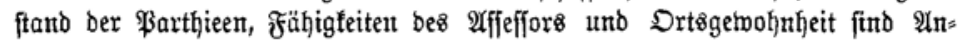
halt\&puntfte. gl. moderando zu c. 11. §. 5. cit. $\mathscr{U} \mathfrak{.}$ einigen Drten maren bie Sportełn ber Rotare fţrirt. gl. not. zu 11. §. 5. cit. 


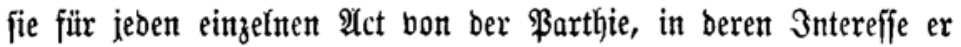
biefen $\mathfrak{A}$ ct borgenommen ${ }^{52}$ ), der 2 (ffeffor bagegen handelt, wie ber

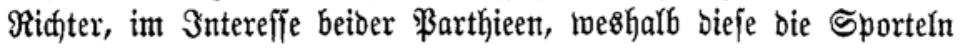
Des Afffeffors zu gleiden Theilen zul tragen haben. ${ }^{53}$ ) Die Armen find ohne 3rweifel aud hier (portelfrei. ${ }^{54}$ )

Beim Beginn dez Broceffez fordert der $\Re$ idjter bon ben

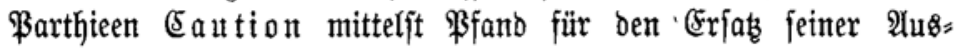
lagen ${ }^{55}$ ), fomie für bie 3ahlung ber Sporteln ber 2 (ffefforen ${ }^{56}$ ), Notare uno übrigen Sierichteperjonen. ${ }^{57}$ )

Welches Berfahren mag eingetreten fein, wenn eine bezm. beibe Barthieen arm zu fein befaupteten? Das Befeb fagt nur,

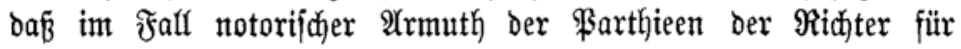
jeine Expenfen nidts forbern folfe. ${ }^{58}$ ) Wurbe bon ben notorija

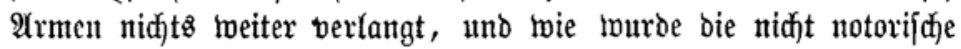

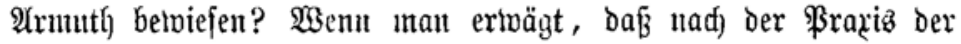

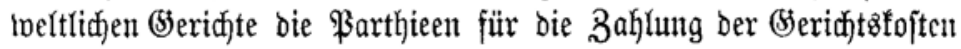
Realcaution fteflen, und im Faff ifres Hubermögens den (Fid ber 2(rmuth Yeifteten und das eiolidfe Beripredjen ber Sporteln= uno Expenfenzahlung bei fünftigen befferen $\mathfrak{B}$ ermögensoberhältniffen $a b=$

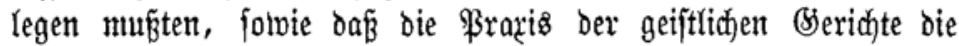

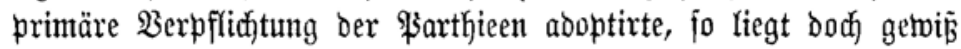

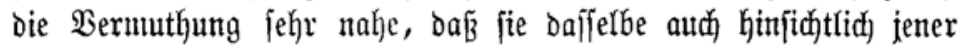
ebentutellen $\mathfrak{B}$ erpfflidututg oer \$arthieen that.

s2) gl. officium зи c. 11. §. 6. cit.

s3) c. 11. §. 5. cit. gl. communiter hierzu.

s4) Der Berpflichtung ber apparitores unb scriptores zur unentgert= lichen Dienftleiftung jür $\mathfrak{A}$ rme gedentt Durantis a. a. $\mathfrak{D}$. de salariis. n. 8 in $\mathrm{f}$.

ss) Tancredus, a. a. D. p. 2. t. 15. §. 2. S. 176. Durantis, a. a. D. n. 10. I. 2. p 1. de satisdationibus. §. 2. n. 3. Эu ber \$rari nahm man hüufig, gerabe wie in ber Braçis ber weltliđen Beridte, Spor=

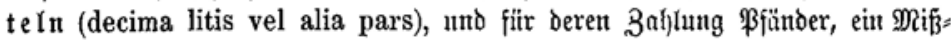
brautc), ber in c. 10. X. de vit. et hon. cler. (3. 1.) gerügt trirb.

s6) Tancredus, a. a. D. Durantis, a. a. D. unb l. 1. p. 1 de assessore $\S .5$ pr. n. 2 . c. 10 . X. cit.

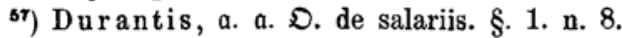

${ }^{38}$ ) c. 11. §. 4. de rescr. in VIto. 


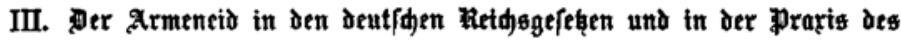 Reidgkammergeridts.}

\section{§. 3.}

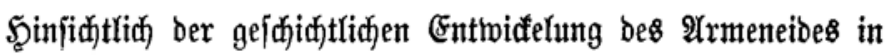

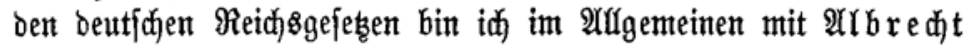

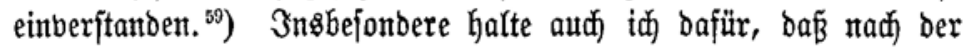
Sammergeridftioronung bon $1555^{60}$ ) $\Im \mathrm{eb} e r$, ber zum $\mathfrak{A} r m e n t e d t$ zugelaffen zu merben berlangt,

1) feine $\mathfrak{A}$ rmuth befdeinigen müffe, und ztwar

a. zumädffit mit einem bon feiner Sbrigfeit ausigeftefllten $\mathfrak{A} r=$ muthszeugniffe; in beffen Ermangelung

b. mit einem bon glaubmürbigen Berjonen ausgejtefliten $\mathfrak{A} r=$ muth 8 zeugniffe, unt in deffen Ermangelung

c. mit andern Mitteln, und

2) ben boflen $\mathfrak{A r m e n t e i ~}^{61}$ ) fóbören müffe. ${ }^{62}$ )

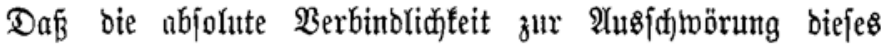

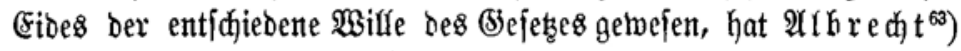
mitteljt des $\mathfrak{B i j i t a t i o n z a b j d j i e \delta s ~ b o n ~} 1713$ §. 110 bargethan.

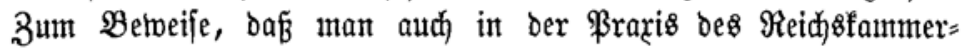

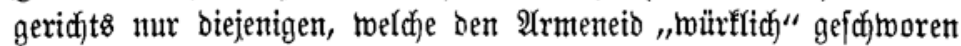

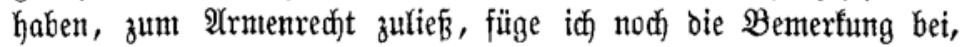

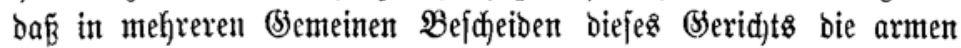
Barthieen immer, "gefdivorene arme Farthicen" genaunt lwerben. ${ }^{\text {. }}$ )

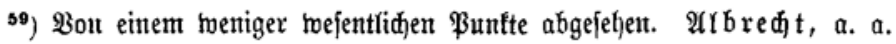
D. S. 94 jđeint bie forberung bes in ber (5. (3. D. bon 1495 §. 24 ant=

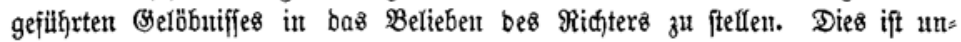

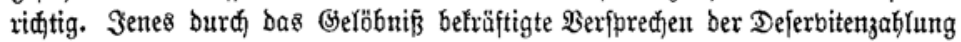
ift eine juratorifdje Caution. Sie zlt verlangen ober nidjt fteht im 2 Biffen ber

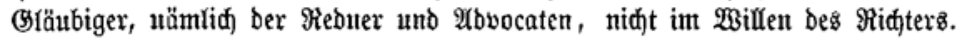

60) Th. 1. T. 41. §. 1.

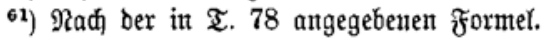

62) MIbređt, a. a. D. S. 100-115.

$\left.{ }^{63}\right)$ a. a. D. S. 121.

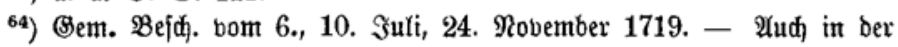

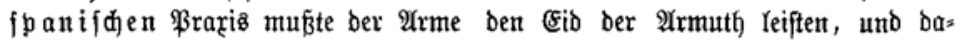

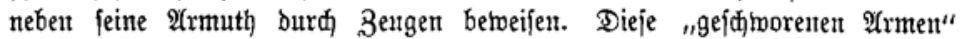
murben ,pobres de solennidad" ,pauperes solemnitatis" genaunt. cf. Salgado de Samoza, labyr. credit. p. 1. c. fin. n. 110-116. 


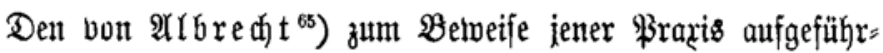

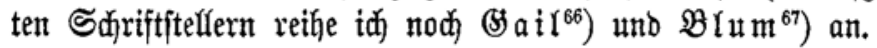

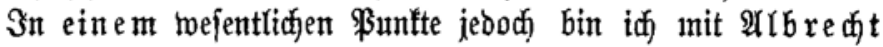
nidift einberjtanden.

(58) handelt fich um bie Snterpretation des

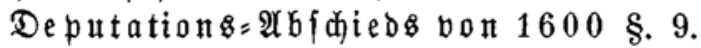

- So jtatuiren, feşen uno orbnen twir, ba hinfüro einer anfeng= lid bie armut flaget, und in mangel ber obangebeuten bocumentorum barein zu fdimeren begert, baß er aljo mit ernjt umb getwififen berifgt ber fachen gefragt, uno bahin an= gehalten merbe, feine Filage burdy einen 2lobocaten ordentlid ftellen,

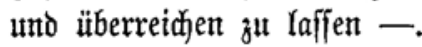

Einige Redft8lehrer faffen bie Stelle, in mangel ber obans gedeuten Documentorum barein zu fdiweren begert" fo auf, als

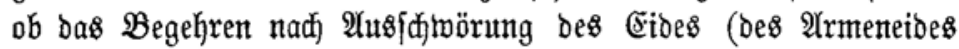

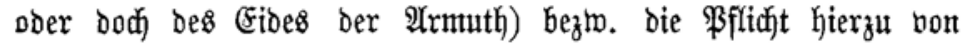

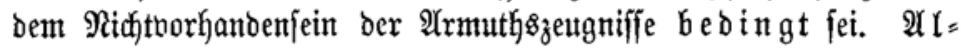

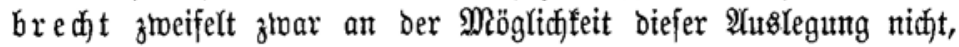
hält aber bod) auth biejenige für ftatthaft, und tregen ihrer Heber. eimftimmuntg mit ber früheren Bsefezgebung für bie twahrffdeinlidjere, monach bie Worte "in mangel ber obangedeuten bocumentorum" greidjbebenteno feien mit: "ber inneren Mangethaftigfeit wirf=

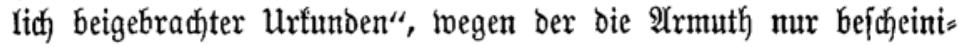

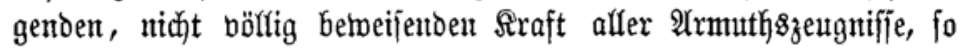

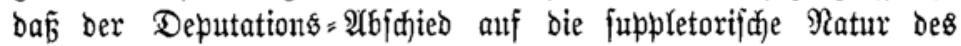
(Eibes habe aufmerffam madjen mollen. Dieje einigermaßen bes

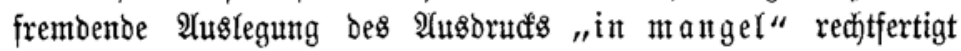

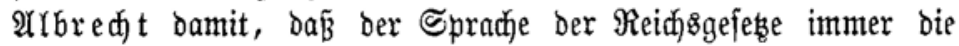
rateinifdie Diction mittelatterlidjer Battung mejentlid) zut (5rande

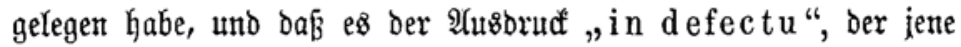
innere Miangelfaftigfeit bezeidnte, getwejent wäre, weldjer dem $\mathfrak{B}$ ortc ,in mangel" entiprädje.

6s) a. a. S. S. $122 . \Re .2$.

$\left.{ }^{66}\right)$ Observ. 1. 1. obs. 43. n. 18.

$\left.{ }^{67}\right)$ Proc. cameral. tit. 64. de supplicat. n. 22. 24. 27. 31. 35.37 seq. Arm triro berienige genamt, qui se tantum in bonis non habere, de quo suis Advocatis, Procuratoribus atque Cancellariae pro labore satisfacere posset, juravit. 


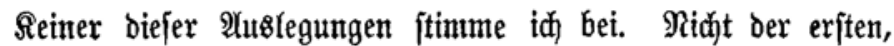

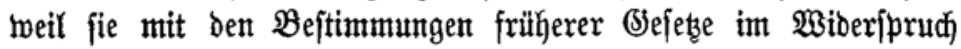
jteht, ohne baß̉ bie Faffung ber betreffenden Stelle einen Saluz

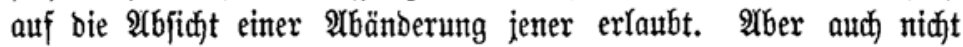
Der $\mathfrak{A r b r e d} t^{\prime}$ fden. Denn in ber Befdidjte erfdeint Der Eib

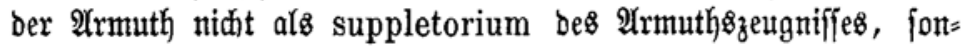

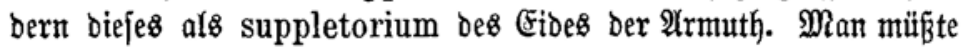

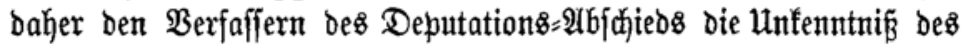
Armeneibes zum Bortwurf machen, wozu, zumal wegen ber Sürze

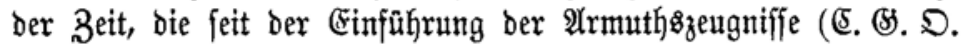

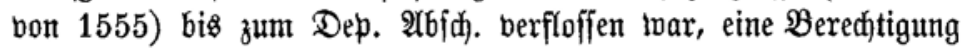
nididt borliegt.

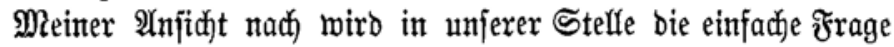
entjdieden, wie es ju halten jei, ment eine \$arthie arm fu fein

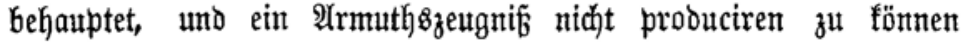

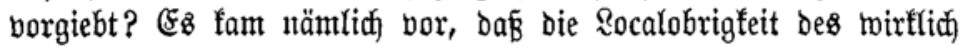

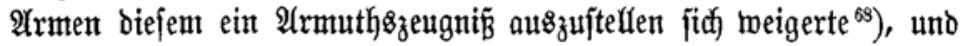

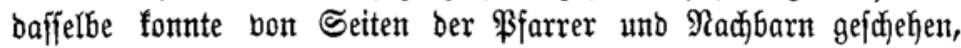
twie es aud borfonmen fonnte, Daß̧ twegen ber Domicillofitgfeit eines

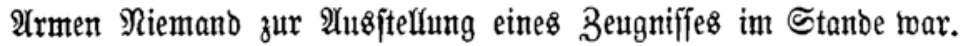

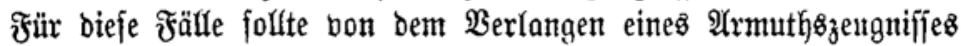
abgejtanden, und ber angeblid 2rme ohne Weiteres zum 2Yrmeneibe

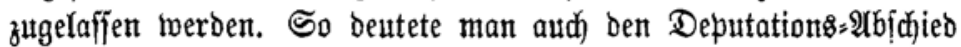

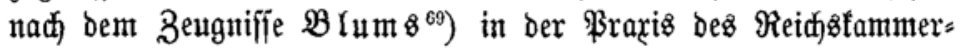
gerichts.

68) Brum, a. a. D. n. 24.

$\left.{ }^{69}\right)$ a. a. D. n. 26. Bon einer anderen Deutung ift hier nidjt bie ßebe. 